\title{
First Doppler imaging of the RS CVn binary FF UMa
}

\author{
H.V.Şenavcı, E. Bahar, İ. Özavcı and T. Kılı̧̧oğlu \\ Department of Astronomy and Space Sciences, Faculty of Science, Ankara \\ University, Tandoğan 06100 Ankara, Turkey
}

Received: October 23, 2019; Accepted: January 8, 2020

\begin{abstract}
We present the first Doppler Imaging (DI) study of the chromospherically active RS CVn binary FF Ursae Majoris. New mid-resolution $(R \sim 13500)$ time series spectral data were acquired using the echelle spectrograph attached to the 0.4-m Kreiken Telescope at the Ankara University Kreiken Observatory. We applied a spectral subtraction technique to the $\mathrm{H}_{\alpha}$ line to reveal the phase behavior of the chromospheric activity of the system. The results derived from DI and spectral subtraction analyses show that both components exhibit photospheric and chromospheric activity. The time series spectral data also enable us to obtain the most recent radial velocity curve of the system and re-determine the physical parameters.
\end{abstract}

Key words: stars: activity stars: imaging

\section{Introduction}

Understanding the underlying astropyhsical processes of magnetic activity in binary stars is complicated, especially due to tidal interactions that significantly affect the mechanisms responsible for stellar activity. In this context, RS CVntype binaries are crucial laboratories to reveal the effects of tidal interaction, for instance, surface differential rotation (Kővári et al., 2017). Among the methods used to investigate stellar magnetic activity, Doppler imaging (DI) is a powerful and widely used technique that allows us to obtain stellar spot distributions by means of spot filling factors or temperature maps. Signal enhancing techniques like LSD (Donati et al., 1997) provide a higher SNR of spectral lines and hence more precise surface maps, even with the lower resolution. In this study, we performed the first DI study of the RS CVn-type binary FF UMa and we used the spectral synthesis method to investigate the activity nature of both components of the system. We also obtained the most recent orbital parameters of FF UMa with the help of the radial velocity analysis of the spectral data.

The activity nature of FF UMa was first identified by Pounds et al. (1993) using the ROSAT Wide Field Camera all-sky survey. Several studies concerning the system in the literature (Jeffries et al., 1995; Henry et al., 1995; Fekel, 1997; Strassmeier et al., 2000; Griffin, 2012) investigate determinations of orbital parameters, $v \sin i$, spectral types, as well as estimates of masses and radii, 
since there is no information on the orbital inclination due to the non-eclipsing geometry of the system. The most detailed activity-related study of FF UMa was performed by Gálvez et al. (2007), who carried out an extensive study of the optical indicators of chromospheric activity, revealing that both components are quite active. They also found a remarkable orbital period variation that is attributed to the presence of magnetic activity. Another detailed study by Strassmeier et al. (2012) with high-precision photometry and spectroscopy clearly showed that both components are subgiants showing significant amounts of activity. The above results from the literature together with a lack of any DI study of the RS CVn-type binary FF UMa in the literature led us to investigate the system.

\section{Observations and data reduction}

We observed the system spectroscopically using the mid-resolution echelle spectrograph attached to the $0.4 \mathrm{~m}$ Kreiken Telescope at the Ankara University Kreiken Observatory. The average resolution of the spectrograph is $R \sim 13500$ and the wavelength range is between 4340 and $7400 \AA$. We gathered 17 spectra within 4 months of observing run, with an exposure time of 3600 seconds and a signal-to-noise ratio (SNR) between 43 and 76 . The current spectral resolution together with the average $v \sin i$ values gave us a spot resolution of $\sim 27^{\circ}$. We also observed standard stars HD 161053 (K7 III), HD 158332 (K1 IV) and HD $182488(\mathrm{~K} 0 \sim \mathrm{V})$ that are required by both the spectral synthesis method and the DI analysis to represent spotted and unspotted photospheres. We used the AudeLA software (Klotz et al., 2012) for data reduction and wavelength calibration and performed normalization using our own code that is developed in Python.

\section{Analysis}

\subsection{Radial velocities and radii}

We used the profiles obtained using the LSD technique by Donati et al. (1997) to determine the radial velocity data points. In this context, with the help of a Python code, we generate synthetic models and adjust the width, depth and the center of the rotational profile using the the nonlinear least-squares minimization Python package lmfit (Newville et al., 2018). The SNR values of the profiles obtained using $\sim 2800$ lines are between 843 and 1094. We used the rvfit code by Iglesias-Marzoa et al. (2015) to determine the orbital parameters and found $q=M_{2} / M_{1}=0.4587 \pm 0.0076, K_{1}=29.217 \pm 0.442 \mathrm{~km} / \mathrm{s}, V_{\gamma}=-2.97 \pm 0.27$ $\mathrm{km} / \mathrm{s}, T_{0}(\mathrm{HJD})=2454067.01947 \pm 0.001689$ and $P=3.27487 \pm 0.00004 \mathrm{~d}$. The rv fits are shown in the left panel of Fig. 1. Since there is no (or negligible) eclipse due to the low orbital inclination of the system, the absolute parameters are limited by $\sin i$. In addition to the orbital inclination, the determination of 
the radii is also crucial for the DI analysis. Therefore, with the help of the $T_{\text {eff }^{-}}$ $\log g$ parameters of the components obtained by Strassmeier et al. (2012), we used pre-main-sequence PARSEC isochrones (Bressan et al., 2012) to determine radii upper and lower limits by considering the uncertainties of the $T_{\text {eff }}$ and $\log g$ parameters given by Strassmeier et al. (2012). We obtained the radii of primary and secondary components as $R_{1}=2.65 \pm 0.79 R_{\odot}$ and $R_{2}=2.17 \pm 0.85 R_{\odot}$, respectively. The isochrones as well as the locations of the components of the system are shown in the right panel of Fig. 1.
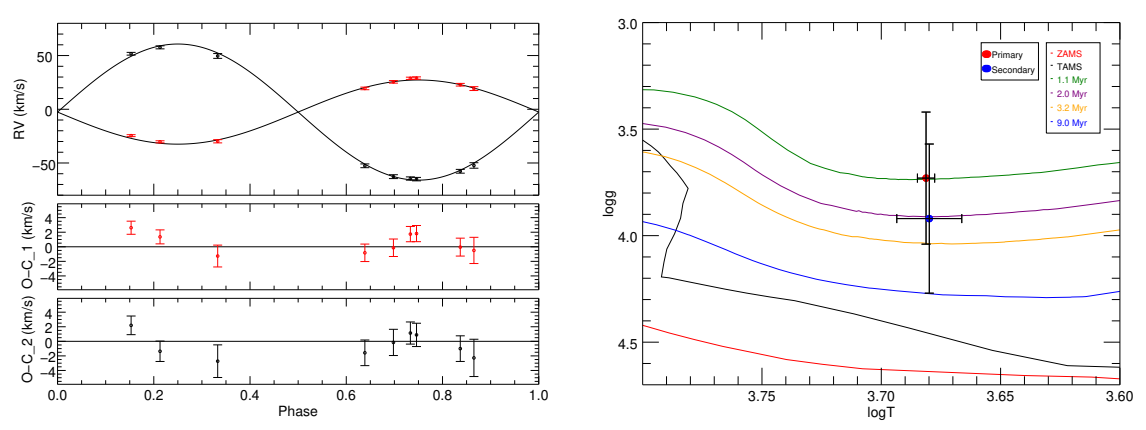

Figure 1. Left panel: RV curve of FF UMa. The red and black open circles represent the RV data of the primary and secondary components, respectively, while the solid black line represents the RV fit to the data. Right panel: Locations of primary and secondary components of FF UMa in a gravity-temperature diagram.

\subsection{Doppler imaging and chromospheric activity}

In order to Doppler image both components of FF UMa, we used the DI code DoTS by Collier Cameron (1997), which performs surface reconstruction by means of spot filling factors based on a two-temperature model using the Maximum Entropy Method (MEM). It is also possible to fine-tune and/or obtain some astrophysical parameters using the DoTS code with the help of a grid search depending on the chi-square minimization. In this context, we performed a multi-dimensional grid search by adjusting the orbital inclination $(i)$, radii $\left(R_{1,2}\right)$ and LSD line strengths of both components $\left(E W_{1,2}\right)$. From the grid search, we obtained an orbital inclination of $i=50.5$, and radii $R_{1}=2.95 R_{\odot}$ and $R_{2}=2.30 R_{\odot}$. The resultant surface reconstructions of both components as Mercator projections are given in Fig. 2.

To study the chromospheric activity behavior of FF UMa and search for a possible correlation with the photospheric activity, we applied the spectral subtraction technique using the $\mathrm{H}_{\alpha}$ line to look for the equivalent width (EQW) variation along with the orbital phase. We used HD 158332 as non-active stan- 

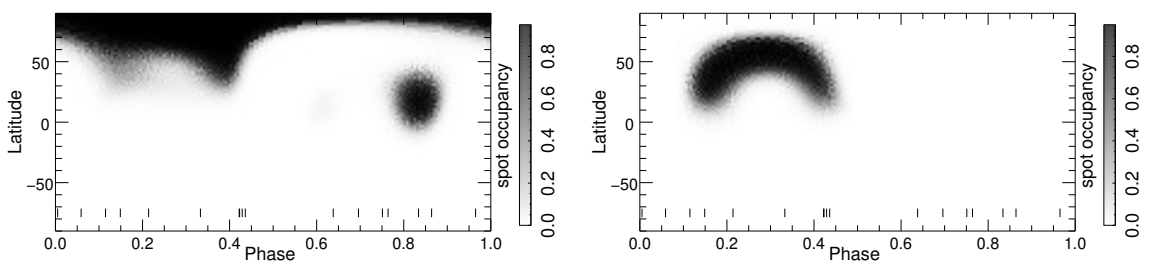

Figure 2. The Mercator projections of the primary (left panel) and the secondary (right panel) components of FF UMa.

dard star and obtained the EQW values using Gaussian fits, and finally plot them versus orbital phase as shown in Fig. 3. As seen from the right panel of Fig. 3, there are two inconsistent points showing high EQW values that may be a consequence of a flare phase (pre- or post-flare). In order to investigate that inconsistency we also looked at the HeI D3 line but due to the low SNR and resolution, we could not clearly distinguish if this line is in emission or not.
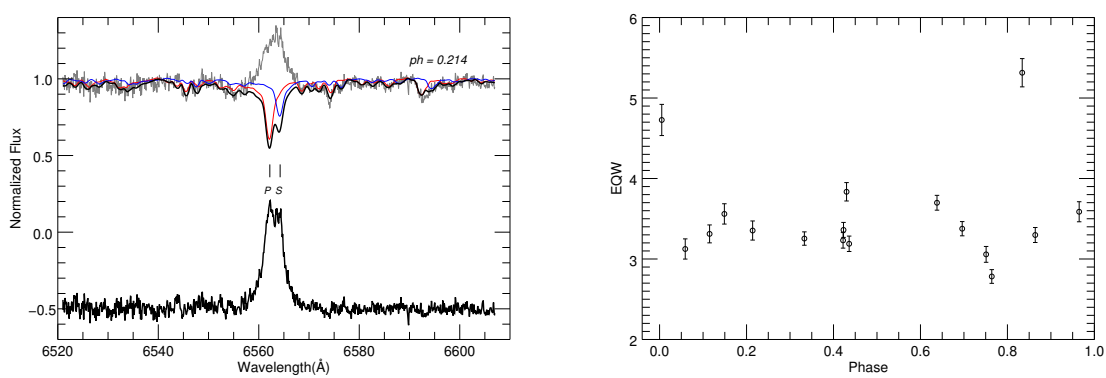

Figure 3. The spectral subtraction of the $H_{\alpha}$ line at phase 0.214 (left panel). The grey solid line represents the normalized spectrum of FF UMa. The red and blue solid lines show the spectra of the primary and secondary components, respectively, while the black solid line represents the total flux. The EQW variation of the system throughout the orbital phase with $3 \sigma$ errors is shown in the right panel.

\section{Discussion and conclusion}

We performed the first Doppler imaging of the RS CVn type binary FF UMa and revealed that both components have high latitude spots, while the primary has a low-to-mid spot feature at around phase 0.8 (see Fig. 2). As also clear from Fig. 2, the spot feature on the secondary is predominant between phases 
0.1 and 0.45 , approximately. We also determined the orbital parameters with the help of the radial velocity curve analysis. The results are consistent with those obtained in the literature.

It is also possible to generate synthetic light curves using the resultant DI map with the code DoTS, which allows us to compare the photospheric and chromospheric behavior of the system. In this context, we generated a $V$-band light curve using the surface reconstructions of both components. After removing two inconsistent points that may be the consequence of a possible flare event, we compared the variation of EQW and the synthetic light curve with the orbital phase as shown in Fig. 4. The similarity between the equivalent width and flux

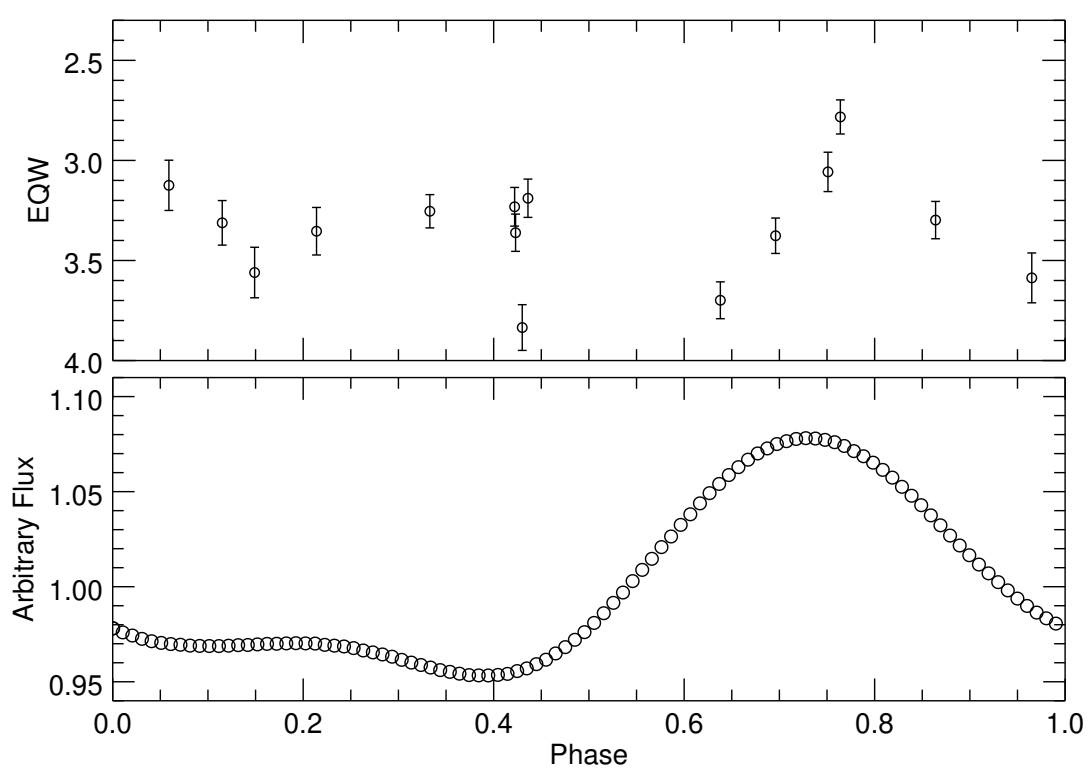

Figure 4. A comparison of EQW variation of the $H_{\alpha}$ line and the sysnthetic light curve obtained from surface reconstructions of both components of FF UMa.

variations is clear from Fig. 4, as the highest flux in the synthetic light curve corresponds to the lowest equivalent width. The overall variations also show similarities. This results also showed that the surface maps obtained in this study are reliable even for the mid-resolution spectral data. The light curve of the system observed by Strassmeier et al. (2012) is also quite similar to that obtained in this study from DI maps, which again shows the reliability of spot distributions from DI. That similarity may also be a consequence of long-lived 
polar spots. Frequent monitoring with higher resolution spectroscopy as well as photometry would enlighten the activity nature of this interesting system.

Acknowledgements. Authors acknowledge the support by The Scientific And Technological Research Council Of Turkey (TÜBİTAK) through the project 1001$115 \mathrm{~F} 033$.

\section{References}

Bressan, A., Marigo, P., Girardi, L., et al., PARSEC: stellar tracks and isochrones with the PAdova and TRieste Stellar Evolution Code. 2012, Mon. Not. R. Astron. Soc., 427, 127, DOI: $10.1111 / \mathrm{j} .1365-2966.2012 .21948 . x$

Collier Cameron, A., Eclipse mapping of late-type close binary stars. 1997, Mon. Not. R. Astron. Soc., 287, 556, DOI: 10.1093/mnras/287.3.556

Donati, J.-F., Semel, M., Carter, B. D., Rees, D. E., \& Collier Cameron, A., Spectropolarimetric observations of active stars. 1997, Mon. Not. R. Astron. Soc., 291, 658, DOI: $10.1093 / \mathrm{mnras} / 291.4 .658$

Fekel, F. C., Rotational Velocities of Late-Type Stars. 1997, Publ. Astron. Soc. Pac., 109, 514, DOI: $10.1086 / 133908$

Gálvez, M. C., Montes, D., Fernández-Figueroa, M. J., de Castro, E., \& Cornide, M., Multiwavelength optical observations of chromospherically active binary systems. V. FF UMa (2RE J0933+624): a system with orbital period variation. 2007, Astron. Astrophys., 472, 587, DOI: 10.1051/0004-6361:20067015

Griffin, R. F., The constant orbital period of FF Ursae Majoris. 2012, Astron. Astrophys., 537, A56, DOI: 10.1051/0004-6361/201116593

Henry, G. W., Fekel, F. C., \& Hall, D. S., An Automated Search for Variability in Chromospherically Active Stars. 1995, Astron. J., 110, 2926, DOI: 10.1086/117740

Iglesias-Marzoa, R., López-Morales, M., \& Jesús Arévalo Morales, M., The rvfit Code: A Detailed Adaptive Simulated Annealing Code for Fitting Binaries and Exoplanets Radial Velocities. 2015, Publ. Astron. Soc. Pac., 127, 567, DOI: 10.1086/682056

Jeffries, R. D., Bertram, D., \& Spurgeon, B. R., New Extreme Ultraviolet-Selected Active Binaries from the ROSAT Wide Field Camera all Sky Survey. 1995, Mon. Not. R. Astron. Soc., 276, 397, DOI: 10.1093/mnras/276.2.397

Kővári, Z., Oláh, K., Kriskovics, L., et al., Rotation-differential rotation relationships for late-type single and binary stars from Doppler imaging. 2017, Astronomische Nachrichten, 338, 903, DOI: 10.1002/asna.201713400

Klotz, A., Delmas, R., Marchais, D., Pujol, M., \& Jasinski, C., The AudeLA software. 2012, in Astronomical Society of India Conference Series, Vol. 7, Astronomical Society of India Conference Series, .15

Newville, M., Otten, R., Nelson, A., et al. 2018, lmfit/lmfit-py 0.9 .12

Pounds, K. A., Allan, D. J., Barber, C., et al., The ROSAT Wide Field Camera allsky survey of extreme-ultraviolet sources. I. The bright source catalogue. 1993, Mon. Not. R. Astron. Soc., 260, 77, DOI: 10.1093/mnras/260.1.77 
Strassmeier, K., Washuettl, A., Granzer, T., Scheck, M., \& Weber, M., The ViennaKPNO search for Doppler-imaging candidate stars. I. A catalog of stellar-activity indicators for 1058 late-type Hipparcos stars. 2000, Astron. Astrophys., Suppl., 142, 275, DOI: 10.1051/aas:2000328

Strassmeier, K. G., Weber, M., Granzer, T., \& Järvinen, S., Rotation, activity, and lithium abundance in cool binary stars. 2012, Astronomische Nachrichten, 333, 663, DOI: $10.1002 /$ asna.201211719 